\title{
A 60-year-old man with chest pain following pacemaker implantation
}

60-year-old man with variLant angina was taking verapamil to prevent coronary spasm. Symptomatic seconddegree atrioventricular block developed, and he required implantation of a permanent dualchamber pacemaker. However, during the implantation procedure, repeated attempts to penetrate the left subclavian and left cephalic veins failed. Subsequent injection of contrast medium revealed venous obstruction with an alternative collateral circulation around the clavicle. The pacing leads were eventually introduced into the right cardiac chambers through the left jugular vein. At the end of the procedure, left chest pain developed. Physical examination and an electrocardiogram revealed normal findings, but a chest radiograph showed the presence of a small area of pneumopericardium (Fig. 1), which resolved spontaneously after 24 hours (Fig. 2) and was $\approx$ felt to be the cause of his pain.

Most patients undergoing pacemaker implantation have some discomfort or ecchymoses at the site of the incision in the early postoperative period, and mild analgesics may be required. Acute complications after implantation are not uncommon, occurring in $4 \%-7 \%$ of cases, and most frequently consist of lead displacement, traumatic pneumothorax, hemopneumothorax and pericardial tamponade.

Pneumopericardium may develop in a number of situations. It may be spontaneous and occur in conjunction with hemopericardium or some type of effusion. Also, it may follow penetrating wounds to the chest or upper abdomen, or wounds or fistulous communications due to primary disease, malignant disease or damage to adjacent viscera by surgical instruments. Pericardial infection by gas-producing organisms can also lead to such a clinical picture. Pericardial complications have been reported because of perforation following insertion of a temporary ventricular endocardial lead. ${ }^{2}$ However, isolated pneumopericardium reported as an exclusive complication of a permanent pacemaker implantation is very uncommon.

The mechanism of the pneumopericardium our patient experienced remains elusive, although we believe it may have been caused by accidental perforation through the wall of the superior vena cava in its intrapericardial tract, allowing migration of air into the pericardium through weak points in the pericardial reflection over the great vessels. However, accidental atrial perforation cannot be completely excluded as a cause of the pneumopericardium, but this is unlikely since the atrial pacing lead's threshold did not show any change. ${ }^{3}$

Alfonso Sestito

Gregory Angelo Sgueglia

Fabio Infusino

Paolo Zecchi

Filippo Crea

Gaetano Antonio Lanza

Institute of Cardiology

Università Cattolica del Sacro Cuore

Rome, Italy

\section{References}

1. Tobin K, Stewart J, Westveer D Frumin H. Acute complications of permanent pacemaker implantation: their financial implication and relation to volume and operator experience. Am 7 Cardiol 2000;85:774-6.

2. Deanfield J, Jonathan A, Fox K. Pericardial complications of endocardial and epicardial pacing. $\mathrm{Br} \mathrm{Med} \mathcal{F}$ (Clin Res $E d)$ 1981;283:635-6.

3. Srivathsan K, Byrne RA, Appleton CP, Scott LRP. Pneumopericardium and pneumothorax contralateral to venous access site after permanent pacemaker implantation. Europace 2003;5:361-3.
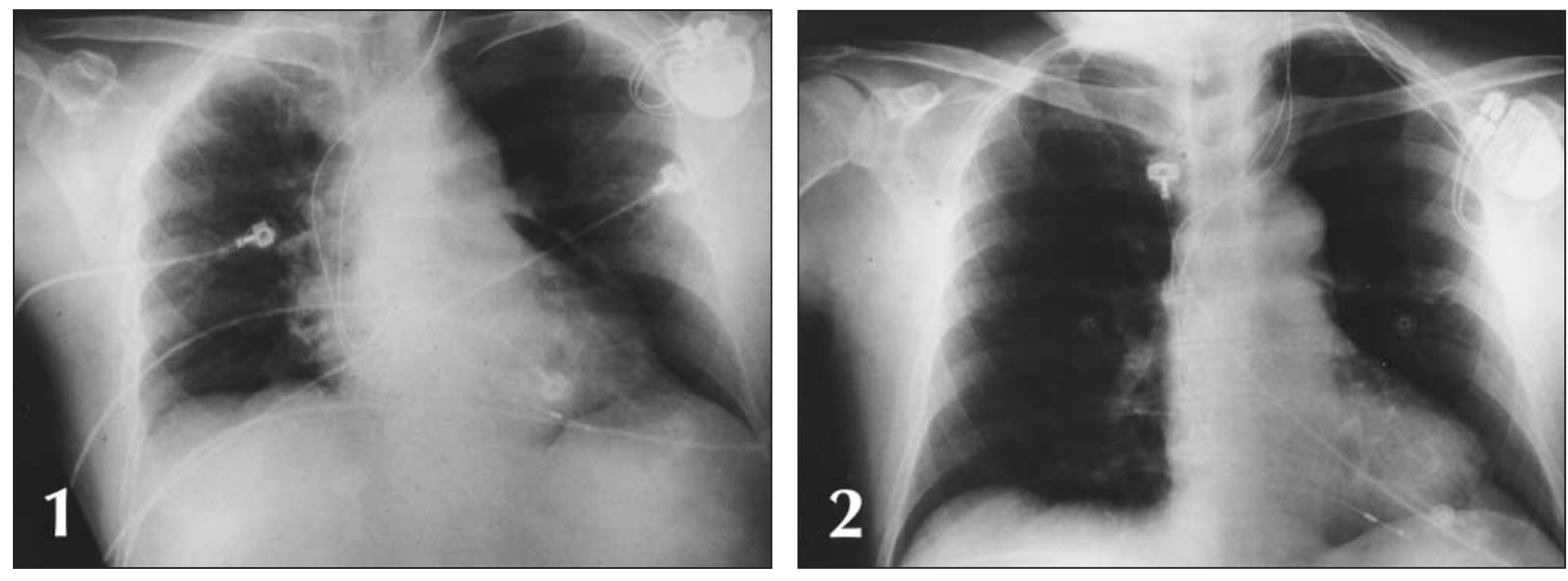